\title{
Inverse eigenvalue problems for centrosymmetric matrices under a central principal submatrix constraint
}

\author{
Li-Jun Zhao a,*, Ru Huang ${ }^{b}$ \\ ${ }^{a}$ School of Mathematics and Information Engineering, Taizhou University, Linhai, Zhejiang, 317000, P. R. China. \\ ${ }^{b}$ Department of Mathematics \& Computer Science, Emory University, Atlanta, Georgia 30322, USA.
}

\begin{abstract}
This article considers an inverse eigenvalue problem for centrosymmetric matrices under a central principal submatrix constraint and the corresponding optimal approximation problem. We first discuss the specified structure of centrosymmetric matrices and their central principal submatrices. Then we give some necessary and sufficient conditions for the solvability of the inverse eigenvalue problem, and we derive an expression for its general solution. Finally, we obtain an expression for the solution to the corresponding optimal approximation problem. (C)2017 All rights reserved.
\end{abstract}

Keywords: Centrosymmetric matrix, central principal submatrix, inverse eigenvalue problem, optimal approximation problem. 2010 MSC: 15A57, 15A18, 65F35.

\section{Introduction}

We first introduce some notations. Let $R^{n \times m}$ denote the set of all $n \times m$ real matrices. Let $O^{n \times n}$ be the set of all $n \times n$ orthogonal matrices. Let $A^{\top}, \operatorname{rank}(A)$ and $A^{+}$represent the transpose, rank and MoorePenrose generalized inverse of matrix $A$, respectively. Let $I_{n}$ denote the identity matrix of order $n$, and 0 be a zero matrix or vector of size implied by context. We use $\langle A, B\rangle=\operatorname{trace}\left(B^{\top} A\right)$ to define the inner product of matrices $A$ and $B$ in $R^{n \times m}$. Then $R^{n \times m}$ is a Hilbert inner product space. The norm of a matrix generated by the inner product is the Frobenius norm $\|\cdot\|$, that is, $\|A\|=\sqrt{\langle A, A\rangle}=\left(\operatorname{trace}\left(A^{\top} A\right)\right)^{\frac{1}{2}}$.

Definition 1.1. A real $n \times n$ matrix $A=\left(a_{i, j}\right)$ is called a centrosymmetric matrix if its elements satisfy the properties

$$
a_{i, j}=a_{n-i+1, n-j+1}, \quad \text { for } 1 \leqslant i, j \leqslant n .
$$

The set of all $n \times n$ centrosymmetric matrices is denoted by $\operatorname{CSR}^{n \times n}$.

Centrosymmetric matrices play an important role in areas such as the numerical solution to certain differential equations [1], various engineering problems [5], and the study of some Markov processes [16].

\footnotetext{
*Corresponding author

Email address: zhaolijun2013@163.com (Li-Jun Zhao)
}

doi:10.22436/jmcs.017.04.04 
In particular, Kimura [12] studied a Markov process whose transition matrix $R$ is an $(n+1) \times(n+1)$ centrosymmetric matrix. The matrix $R$ is given when $\alpha=2$ by

$$
R_{i+1, j+1}=\left(\begin{array}{c}
(n-i) \alpha \\
n-j
\end{array}\right)\left(\begin{array}{c}
i \alpha \\
j
\end{array}\right) /\left(\begin{array}{c}
n \alpha \\
n
\end{array}\right), \quad 0 \leqslant i, j \leqslant n .
$$

In fact, symmetric Toeplitz matrices and persymmetric Hankel matrices are two useful examples of centrosymmetric matrices. Good [8] pointed out that "Toeplitz matrices arise as discrete approximations to kernels $k(x, t)$ of integral equations when these kernels are functions of $|x-t|$. Similarly if a kernel is an even function of its vector argument $(x, t)$, that is $k(x, t)=k(-x,-t)$, then it can be discretely approximated by a centrosymmetric matrix."

Several interesting results are available in the literature on centrosymmetric matrices [14, 20]. In particular, Peng et al. discussed the linear constrained problem of centrosymmetric matrices with a leading principal submatrix constraint [14]. The problem, finding solutions of a matrix equation under a principal submatrix constraint, comes from a practical subsystem expansion problem. Therefore, researchers have great interest in studying a variety of problems under submatrices constraint of late years [6, 14, 17]. Because of the specified structure of centrosymmetric matrices, it is unfit for discussing centrosymmetric matrices under their leading principal submatrices constraint, for it destroys the special symmetric of centrosymmetric matrices. Therefore, we present a different concept, a central principal submatrix, which was first defined in [18]. The definition is as follows.

Definition 1.2. If $n-k$ is even, then the $k$-square central principal submatrix $A_{c}(k)$ of a given matrix $A \in R^{n \times n}$ is a $k$-square submatrix obtained by deleting the first and last $\frac{n-k}{2}$ rows and columns of $A$, that is

$$
A_{c}(k)=\left(0, I_{k}, 0\right) A\left(0, I_{k}, 0\right)^{\top}, \quad 0 \in R^{k \times \frac{n-k}{2}} .
$$

It is intuitive and obvious that a matrix of odd (even) order only has central principal submatrices of odd (even) order.

Throughout this paper, we denote by $A[k]$ the right-bottom $k \times k$ principal submatrix of $A \in R^{n \times n}$, that is

$$
A[k]=\left(a_{i, j}\right)_{i, j=n-k+1}^{n}=\left(0, I_{k}\right) A\left(0, I_{k}\right)^{T} .
$$

In particular, an inverse eigenvalue problem is as follows. Given vectors $x_{1}, \cdots, x_{l}$ and scalars $\lambda_{1}, \cdots, \lambda_{l}$ where $1 \leqslant l<n$, construct an $n \times n$ matrix $A \in S$ such that

$$
A x_{j}=\lambda_{j} x_{j}, j=1, \cdots, l,
$$

where $S$ is a subset of $R^{n \times n}$ with specified structures.

It may arise from the design of Hopfield networks and the mass-spring system [13], in a remarkable variety of applications such as applied mechanics and structure design [11], in the discrete analogue of the inverse Sturm-Liouville problem [19] and in vibration design [15]. Studies of inverse eigenvalue problems have ranged from engineering application to algebraic theorization $[2-4,10,20]$. The inverse eigenvalue problem for centrosymmetric matrices under a central principal submatrix constraint is presented in light of the extended matrix preserves the centrosymmetric structure, thus this problem has practical applications. Furthermore, it allows us to extend the existing solutions. However, it has not been considered yet. In this paper, we will discuss this problem and its optimal approximation. We first discuss the special structure of eigenvalues and eigenvectors for a real matrix in real number field.

If a real matrix $A$ has a complex eigenvalue $\lambda_{j}=\alpha_{j}+i * \beta_{j}\left(\alpha_{j}, \beta_{j}\right.$ are real numbers, $1 \leqslant j \leqslant l$, $i=\sqrt{-1})$, and the associated complex eigenvector is $x_{j}=\xi_{j}+i * \eta_{j}\left(\xi_{j}, \eta_{j}\right.$ are real vectors), then $\bar{\lambda}_{j}=\alpha_{j}-i * \beta_{j}$ is also an eigenvalue of $A$ with the associated eigenvector $\bar{x}_{j}=\xi_{j}-i * \eta_{j}$, and

$$
A\left(\xi_{j}, \eta_{j}\right)=\left(\xi_{j}, \eta_{j}\right)\left(\begin{array}{cc}
\alpha_{j} & \beta_{j} \\
-\beta_{j} & \alpha_{j}
\end{array}\right) .
$$


Thus, if $x_{j}$ is a real eigenvector associated with a real eigenvalue $\lambda_{j}$ of $A$, then let

$$
\widetilde{\Lambda}_{j}=\lambda_{j}, \quad \widetilde{x}_{j}=x_{j} .
$$

If $x_{j}=\xi_{j}+i * \eta_{j}$ is a complex eigenvector associated with a complex eigenvalue $\lambda_{j}=\alpha_{j}+i * \beta_{j}$ of $A$, then set

$$
\widetilde{\Lambda}_{j}=\left(\begin{array}{cc}
\alpha_{j} & \beta_{j} \\
-\beta_{j} & \alpha_{j}
\end{array}\right), \quad \widetilde{X}_{j}=\left(\xi_{j}, \eta_{j}\right) .
$$

Hence, for given eigenvalues $\lambda_{1}, \ldots, \lambda_{l}$ and the associated eigenvectors $x_{1}, \ldots, x_{l}$, write

$$
X=\left(\widetilde{X}_{1}, \cdots, \widetilde{X}_{l}\right) \in R^{n \times m}, \quad \Lambda=\operatorname{diag}\left(\widetilde{\Lambda}_{1}, \cdots, \widetilde{\Lambda}_{l}\right) \in R^{m \times m},
$$

where $m \geqslant l$, then (1.1) is equal to $A X=X \wedge$. Therefore, the problem discussed in this paper can be expressed as follows.

Problem 1.3. Given $X \in R^{n \times m}$ and $\Lambda \in R^{m \times m}$ with the form as in (1.4), $A_{0} \in C S R^{k \times k}$, for $k<n$, find an extended matrix $A \in \operatorname{CSR}^{n \times n}$ such that

$$
A X=X \wedge \text {, and } A_{c}(k)=A_{0} .
$$

Problem 1.4. Given an estimate $\widetilde{A} \in R^{n \times n}$, find a matrix $A^{*} \in S_{A}$ such that

$$
\left\|A^{*}-\widetilde{A}\right\|=\min _{\forall A \in S_{A}}\|A-\widetilde{A}\|,
$$

where $S_{A}$ is the solution set of Problem 1.3.

This paper is organized as follows. First, we discuss the specified properties and structure of centrosymmetric matrices and their central principal submatrices. Next, we study eigenvalues and eigenvectors of centrosymmetric matrices, and obtain that the eigenvectors of a centrosymmetric matrix can be expressed in a special form. After that, we convert Problem 1.3 into two inverse eigenvalue problems of half-sized independent real matrices under themselves right-bottom principal submatrices constraint trickily, which is a special feature of this paper. Meanwhile, this simplifies and is crucial to solve Problem 1.3. Later on, we derive the solvability conditions of Problem 1.3 and an expression for its general solution. Finally, we prove that Problem 1.4 has a unique solution and give an expression for it.

\section{The properties of centrosymmetric matrices and their central principal submatrices}

Denote by $e_{i}$ the $i$-th $(i=1,2, \cdots, n)$ column of $I_{n}$, and let $S_{n}=\left(e_{n}, e_{n-1}, \cdots, e_{1}\right)$, then

$$
S_{n}=S_{n}^{\top}, S_{n} S_{n}^{\top}=I_{n} .
$$

Lemma 2.1 ([7]). A matrix $A \in \operatorname{CSR}^{n \times n}$ if and only if $S_{n} A S_{n}=A$.

Let $r=\left[\frac{\mathfrak{n}}{2}\right]$, where $\left[\frac{\mathfrak{n}}{2}\right]$ is the maximum integer which is not greater than $\frac{\mathfrak{n}}{2}$. And let

$$
D_{n}=\frac{1}{\sqrt{2}}\left(\begin{array}{cc}
I_{r} & I_{r} \\
S_{r} & -S_{r}
\end{array}\right)(n=2 r), \quad D_{n}=\frac{1}{\sqrt{2}}\left(\begin{array}{ccc}
I_{r} & 0 & I_{r} \\
0 & \sqrt{2} & 0 \\
S_{r} & 0 & -S_{r}
\end{array}\right)(n=2 r+1) .
$$

It is easy verified that the above matrices $D_{n}$ are orthogonal matrices.

Lemma 2.2 ([20]). $A \in C^{2 r \times 2 r}$ if and only if there exist $M, N \in R^{r \times r}$ such that

$$
A=\left(\begin{array}{cc}
M & N S_{r} \\
S_{r} N & S_{r} M S_{r}
\end{array}\right)=D_{2 r}\left(\begin{array}{cc}
M+N & 0 \\
0 & M-N
\end{array}\right) D_{2 r}^{T} .
$$

$A \in \operatorname{CSR}^{(2 r+1) \times(2 r+1)}$ if and only if there exist $M, N \in R^{r \times r}, u, v \in R^{r}$ and $\alpha \in R$ such that 


$$
A=\left(\begin{array}{ccc}
M & u & N S_{r} \\
v^{\top} & \alpha & v^{\top} S_{r} \\
S_{r} N & S_{r} u & S_{r} M S_{r}
\end{array}\right)=D_{2 r+1}\left(\begin{array}{ccc}
M+N & \sqrt{2} u & 0 \\
\sqrt{2} v^{\top} & \alpha & 0 \\
0 & 0 & M-N
\end{array}\right) D_{2 r+1}^{\top}
$$

Furthermore, when $\mathrm{n}=2 \mathrm{r}$, let

$$
A_{11}=M+N, \quad A_{22}=M-N,
$$

and when $\mathrm{n}=2 \mathrm{r}+1$, set

$$
A_{11}=\left(\begin{array}{cc}
M+N & \sqrt{2} u \\
\sqrt{2} v^{\top} & \alpha
\end{array}\right), \quad A_{22}=M-N,
$$

then $\mathrm{A} \in \mathrm{CSR}^{\mathrm{n} \times \mathrm{n}}$ if and only if there exist $\mathrm{A}_{11} \in \mathrm{R}^{(\mathrm{n}-\mathrm{r}) \times(\mathrm{n}-\mathrm{r})}$ and $\mathrm{A}_{22} \in \mathrm{R}^{\mathrm{r} \times \mathrm{r}}$, whether $\mathrm{n}$ is odd or even, such that

$$
A=D_{n}\left(\begin{array}{cc}
A_{11} & 0 \\
0 & A_{22}
\end{array}\right) D_{n}^{T}
$$

Now we give the special properties of the k-central principal submatrix of a centrosymmetric matrix, that is the submatrix having the same symmetric properties and structure as the given centrosymmetric matrix. Hence they have similar expressions, which is crucial to solve Problem 1.3, for it provides a reasoned way to convert Problem 1.3 to two inverse eigenvalue problems of half-sized independent real matrices under themselves right-bottom principal submatrices constraint. Here, we always assume that $\mathrm{t}=\left[\frac{\mathrm{k}}{2}\right]$.

Lemma 2.3. Let $\mathrm{A} \in \mathrm{CSR}^{\mathrm{n} \times \mathrm{n}}$ have the form as in (2.5). Then the $\mathrm{k}$-square central principal submatrix of $\mathrm{A}$ can be expressed as

$$
A_{c}(k)=D_{k}\left(\begin{array}{cc}
A_{11}[k-t] & 0 \\
0 & A_{22}[t]
\end{array}\right) D_{k}^{T} .
$$

Proof. When $n=2 r$, from (2.1) and the properties of central principal submatrices, that is, a matrix of even order only having central principal submatrices of even order, we have $k=2 t$, and

$$
A_{c}(k)=\left(\begin{array}{cc}
M[t] & N[t] S_{t} \\
S_{t} N[t] & S_{t} M[t] S_{t}
\end{array}\right) .
$$

Thus,

$$
\begin{aligned}
D_{k}^{\top} A_{c}(k) D_{k} & =\frac{1}{\sqrt{2}}\left(\begin{array}{cc}
I_{t} & S_{t} \\
I_{t} & -S_{t}
\end{array}\right) \cdot\left(\begin{array}{cc}
M[t] & N[t] S_{t} \\
S_{t} N[t] & S_{t} M[t] S_{t}
\end{array}\right) \cdot \frac{1}{\sqrt{2}}\left(\begin{array}{cc}
I_{t} & I_{t} \\
S_{t} & -S_{t}
\end{array}\right) \\
& =\left(\begin{array}{cc}
M[t]+N[t] & 0 \\
0 & M[t]-N[t]
\end{array}\right),
\end{aligned}
$$

and (2.3) implies $M[t]+N[t]=A_{11}[t]$ and $M[t]-N[t]=A_{22}[t]$. It says that the $k$-square central principal submatrix of $A$ may be expressed as

$$
A_{c}(k)=D_{k}\left(\begin{array}{cc}
A_{11}[t] & 0 \\
0 & A_{22}[t]
\end{array}\right) D_{k}^{T}
$$

When $n=2 r+1$, from (2.2) and the properties of central principal submatrices, that is, a matrix of odd order only having central principal submatrices of odd order, we have $k=2 t+1$, and

$$
A_{c}(k)=\left(\begin{array}{ccc}
M[t] & u_{t} & N[t] S_{t} \\
v_{t}^{T} & \alpha & v_{t}^{T} S_{t} \\
S_{t} N[t] & S_{t} u_{t} & S_{t} M[t] S_{t}
\end{array}\right), \quad u_{t}=\left(0, I_{t}\right) u, \quad v_{t}=\left(0, I_{t}\right) v
$$

Hence,

$$
D_{k}^{\top} A_{c}(k) D_{k}=\frac{1}{\sqrt{2}}\left(\begin{array}{ccc}
I_{t} & 0 & S_{t} \\
0 & \sqrt{2} & 0 \\
I_{t} & 0 & -S_{t}
\end{array}\right) \cdot\left(\begin{array}{ccc}
M[t] & u_{t} & N[t] S_{t} \\
v_{t}^{\top} & \alpha & v_{t}^{\top} S_{t} \\
S_{t} N[t] & S_{t} u_{t} & S_{t} M[t] S_{t}
\end{array}\right) \cdot \frac{1}{\sqrt{2}}\left(\begin{array}{ccc}
I_{t} & 0 & I_{t} \\
0 & \sqrt{2} & 0 \\
S_{t} & 0 & -S_{t}
\end{array}\right)
$$




$$
=\left(\begin{array}{ccc}
M[t]+N[t] & \sqrt{2} u_{t} & 0 \\
\sqrt{2} v_{t}^{\top} & \alpha & 0 \\
0 & 0 & M[t]-N[t]
\end{array}\right),
$$

and (2.4) implies $\left(\begin{array}{cc}M[t]+N[t] & \sqrt{2} u_{t} \\ \sqrt{2} v_{t}^{\top} & \alpha\end{array}\right)=A_{11}[t+1]$ and $M[t]-N[t]=A_{22}[t]$. It means that the k-square central principal submatrix of $A$ may be written as

$$
A_{c}(k)=D_{k}\left(\begin{array}{cc}
A_{11}[t+1] & 0 \\
0 & A_{22}[t]
\end{array}\right) D_{k}^{\top} .
$$

Combining (2.7) and (2.8), we obtain that the k-square central principal submatrix of $A$ has the form as in (2.6).

It is easy to verify the following lemma from Lemma 2.3.

Lemma 2.4. Suppose that $A \in \operatorname{CSR}^{n \times n}$ has the form as in (2.5). Partition $A_{0} \in \operatorname{CSR}^{k \times k}$ as

$$
A_{0}=D_{k}\left(\begin{array}{cc}
A_{10} & 0 \\
0 & A_{20}
\end{array}\right) D_{k}^{\top}, \quad A_{10} \in R^{(k-t) \times(k-t)}, \quad A_{20} \in R^{t \times t},
$$

then $A_{0}$ is a central principal submatrix of $A$ if and only if $A_{10}=A_{11}[k-t]$ and $A_{20}=A_{22}[t]$.

\section{Expression for the general solution to Problem 1.3}

In this section, we first discuss the properties of eigenvalues and eigenvectors of a centrosymmetric matrix, and obtain that the eigenvectors of a centrosymmetric matrix can be expressed in a special form. Next, utilizing the special expression forms of a centrosymmetric matrix and its central principal submatrices, as discussed in Section 2, we convert Problem 1.3 to two inverse eigenvalue problems of half-sized independent real matrices under themselves right-bottom principal submatrices constraint trickily, which is a special feature of this paper. Finally, we solve Problem 1.3 completely, that is, we provide necessary and sufficient conditions for the existence of a solution to Problem 1.3 and give an expression for the general solution. Now, we investigate the expressions of eigenvectors of a centrosymmetric matrix in the real number field.

Definition 3.1. Let $x \in R^{n}$. $x$ is called a symmetric vector if $S_{n} x=x . x$ is called an anti-symmetric vector if $S_{n} x=-x$.

Given $A \in \operatorname{CSR}^{n \times n}$, if $\lambda_{j}(1 \leqslant j \leqslant l)$ is a real eigenvalue of $A$, and $x_{j}$ is a real eigenvector associated with $\lambda_{j}$, that is $A x_{j}=\lambda_{j} x_{j}$. Then we have, from Lemma 2.1,

$$
A S_{n} x_{j}=S_{n} A x_{j}=\lambda_{j} S_{n} x_{j}
$$

thus $S_{n} x_{j}$ is also an eigenvector of $A$ associated with $\lambda_{j}$. Therefore, $x_{j} \pm S_{n} x_{j}$ are eigenvectors associated with $\lambda_{j}$, where $x_{j}+S_{n} x_{j}$ is a symmetric vector, and $x_{j}-S_{n} x_{j}$ is an anti-symmetric vector.

If $\lambda_{j}=\alpha_{j}+i * \beta_{j}$ is a complex eigenvalue of $A, x_{j}=\xi_{j}+i * \eta_{j}$ is an associated eigenvector, then (1.2) and (1.3) imply $A \widetilde{X}_{j}=\widetilde{X}_{j} \widetilde{\Lambda}_{j}$. From Lemma 2.1, we obtain

$$
A S_{n} \widetilde{X}_{j}=S_{n} A \widetilde{X}_{j}=S_{n} \widetilde{X}_{j} \widetilde{\Lambda}_{j}
$$

thus

$$
A\left(\widetilde{X}_{j} \pm S_{n} \widetilde{X}_{j}\right)=\left(\widetilde{X}_{j} \pm S_{n} \widetilde{X}_{j}\right) \widetilde{\Lambda}_{j}
$$

where the columns of $\widetilde{X}_{j}+S_{n} \widetilde{X}_{j}=\left(\xi_{j}+S_{n} \xi_{j}, \eta_{j}+S_{n} \eta_{j}\right)$ are symmetric vectors, and the columns of $\widetilde{X}_{j}-S_{n} \widetilde{X}_{j}=\left(\xi_{j}-S_{n} \xi_{j}, \eta_{j}-S_{n} \eta_{j}\right)$ are anti-symmetric vectors. 
According to the previous analysis, without loss of generality, we may suppose that $X$ and $\wedge$ have the following forms in Problem 1.3,

$$
\begin{gathered}
X=\left(\begin{array}{cc}
Z_{1} & Y_{1} \\
S_{r} Z_{1} & -S_{r} Y_{1}
\end{array}\right)(n=2 r), \quad X=\left(\begin{array}{cc}
Z_{1} & Y_{1} \\
\sqrt{2} c^{\top} & 0 \\
S_{r} Z_{1} & -S_{r} Y_{1}
\end{array}\right)(n=2 r+1), \\
\Lambda=\operatorname{diag}\left(\Lambda_{1}, \Lambda_{2}\right), \quad \Lambda_{1} \in R^{s \times s}, \quad \Lambda_{2} \in R^{(m-s) \times(m-s)},
\end{gathered}
$$

where $Z_{1} \in R^{r \times s}, Y_{1} \in R^{r \times(m-s)}, c \in R^{s}$, and $\Lambda_{1}, \Lambda_{2}$ are block diagonal matrices, for the block is a square matrix of order 1 or 2 . Then $D_{n}^{\top} X$ has the following form:

$$
\begin{aligned}
& D_{n}^{\top} X=\frac{1}{\sqrt{2}}\left(\begin{array}{cc}
I_{r} & S_{r} \\
I_{r} & -S_{r}
\end{array}\right) \cdot\left(\begin{array}{cc}
Z_{1} & Y_{1} \\
S_{r} Z_{1} & -S_{r} Y_{1}
\end{array}\right)=\left(\begin{array}{cc}
\sqrt{2} Z_{1} & 0 \\
0 & \sqrt{2} Y_{1}
\end{array}\right)(n=2 r), \\
& D_{n}^{\top} X=\frac{1}{\sqrt{2}}\left(\begin{array}{ccc}
I_{r} & 0 & S_{r} \\
0 & \sqrt{2} & 0 \\
I_{r} & 0 & -S_{r}
\end{array}\right) \cdot\left(\begin{array}{cc}
Z_{1} & Y_{1} \\
\sqrt{2} c^{\top} & 0 \\
S_{r} Z_{1} & -S_{r} Y_{1}
\end{array}\right)=\left(\begin{array}{cc}
\sqrt{2} Z_{1} & 0 \\
\sqrt{2} c^{\top} & 0 \\
0 & \sqrt{2} Y_{1}
\end{array}\right)(n=2 r+1) .
\end{aligned}
$$

If $n=2 r$, let $X_{1}=Z_{1}$, and if $n=2 r+1$, let $X_{1}=\left(\begin{array}{l}Z_{1} \\ c^{\top}\end{array}\right)$, then for all arbitrary $n, D_{n}^{\top} X$ may be written as

$$
D_{n}^{\top} X=\left(\begin{array}{cc}
\sqrt{2} X_{1} & 0 \\
0 & \sqrt{2} Y_{1}
\end{array}\right), \quad X_{1} \in R^{(n-r) \times s}, \quad Y_{1} \in R^{r \times(m-s)} .
$$

Lemma 3.2 ([10]). Let $X \in R^{n \times m}$ and $\Lambda \in R^{m \times m}$ as in (1.4), then there exists a matrix $A \in R^{n \times n}$ such that $\mathrm{AX}=\mathrm{X} \wedge$ if and only if $\mathrm{X} \wedge \mathrm{X}^{+} \mathrm{X}=\mathrm{X} \wedge$. Moreover, its general solution can be expressed as $\mathrm{A}=\mathrm{X} \wedge \mathrm{X}^{+}+\mathrm{G}\left(\mathrm{I}_{\mathrm{n}}-\right.$ $\mathrm{XX}^{+}$), where $\mathrm{G} \in \mathrm{R}^{\mathrm{n} \times \mathrm{n}}$ is arbitrary.

Lemma 3.3 ([9]). Given $\mathrm{Y} \in \mathrm{R}^{\mathrm{k} \times \mathrm{n}}, \mathrm{X} \in \mathrm{R}^{\mathrm{m} \times \mathrm{l}}$ and $\mathrm{B} \in \mathrm{R}^{\mathrm{k} \times \mathrm{l}}$, denote

$$
S_{1} \equiv\left\{A \in R^{n \times m} \mid f_{1}(A)=\|Y A X-B\|=\min \right\},
$$

then every element in $\mathrm{S}_{1}$ has the form as

$$
\mathrm{A}=\mathrm{Y}^{+} \mathrm{BX} X^{+}+\mathrm{G}-\mathrm{Y}^{+} \mathrm{YGX} X^{+}, \quad \forall \mathrm{G} \in \mathrm{R}^{\mathrm{n} \times \mathrm{m}} .
$$

In particular, $\mathrm{f}_{1}(\mathrm{~A})=0$ has solutions in $\mathrm{R}^{\mathrm{n} \times \mathrm{m}}$ if and only if $\mathrm{YY}^{+} \mathrm{BX}+\mathrm{X}=\mathrm{B}$, and the general solution has the same form as in (3.4).

We can obtain the following lemma easily from Lemma 3.3.

Lemma 3.4. Given $X, B \in R^{m \times l}$, denote $S_{2} \equiv\left\{A \in R^{n \times m} \mid f_{2}(A)=\|A X-B\|=\min \right\}$, then every element in $\mathrm{S}_{2}$ has the form as

$$
\mathrm{A}=\mathrm{B} \mathrm{X}^{+}+\mathrm{G}\left(\mathrm{I}_{\mathrm{m}}-\mathrm{X} \mathrm{X}^{+}\right), \quad \forall \mathrm{G} \in \mathrm{R}^{\mathrm{n} \times \mathrm{m}} .
$$

In particular, $\mathrm{f}_{2}(\mathrm{~A})=0$ has solutions in $\mathrm{R}^{\mathrm{n} \times \mathrm{m}}$ if and only if $\mathrm{BX}+\mathrm{X}=\mathrm{B}$, and the general solution has the same form as in (3.5).

Theorem 3.5. Given $X \in R^{n \times m}$ as in (3.1), $\Lambda \in R^{m \times m}$ as in (3.2), partition $D_{n}^{\top} X$ as in (3.3). Given $A_{0} \in$ $\operatorname{CSR}^{\mathrm{k} \times \mathrm{k}}$, partition $\mathrm{A}_{0}$ as in (2.9). Set

$$
\begin{aligned}
& Z_{1}=\left(I_{n-r}-X_{1} X_{1}^{+}\right)\left(0, I_{k-t}\right)^{\top}, \quad K_{1}=A_{10}-\left(0, I_{k-t}\right) X_{1} \Lambda_{1} X_{1}^{+}\left(0, I_{k-t}\right)^{\top}, \\
& Z_{2}=\left(I_{r}-Y_{1} Y_{1}^{+}\right)\left(0, I_{t}\right)^{\top}, \quad K_{2}=A_{20}-\left(0, I_{t}\right) Y_{1} \Lambda_{2} Y_{1}^{+}\left(0, I_{t}\right)^{\top} .
\end{aligned}
$$


Problem 1.3 is solvable if and only if

$$
\begin{gathered}
X_{1} \Lambda_{1} X_{1}^{+} X_{1}=X_{1} \Lambda_{1}, \quad Y_{1} \Lambda_{2} Y_{1}^{+} Y_{1}=Y_{1} \Lambda_{2} \\
K_{1} Z_{1}^{+} Z_{1}=K_{1}, \quad K_{2} Z_{2}^{+} Z_{2}=K_{2} .
\end{gathered}
$$

Furthermore, every matrix $A$ in the solution set $S_{A}$ may be written as

$$
A=D_{n}\left(\begin{array}{cc}
X_{1} \wedge_{1} X_{1}^{+}+G_{1}\left(I_{n-r}-X_{1} X_{1}^{+}\right) & 0 \\
0 & Y_{1} \wedge_{2} Y_{1}^{+}+G_{2}\left(I_{r}-Y_{1} Y_{1}^{+}\right)
\end{array}\right) D_{n}^{\top},
$$

where

$$
G_{1}=\left(\begin{array}{c}
E_{1} \\
K_{1} Z_{1}^{+}+E_{2}\left(I_{n-r}-Z_{1} Z_{1}^{+}\right)
\end{array}\right), \quad G_{2}=\left(\begin{array}{c}
F_{1} \\
K_{2} Z_{2}^{+}+F_{2}\left(I_{r}-Z_{2} Z_{2}^{+}\right)
\end{array}\right),
$$

where $\mathrm{E}_{1} \in \mathrm{R}^{(\mathrm{n}-\mathrm{r}-\mathrm{k}+\mathrm{t}) \times(\mathrm{n}-\mathrm{r})}, \mathrm{E}_{2} \in \mathrm{R}^{(\mathrm{k}-\mathrm{t}) \times(\mathrm{n}-\mathrm{r})}, \mathrm{F}_{1} \in \mathrm{R}^{(\mathrm{r}-\mathrm{t}) \times \mathrm{r}}$ and $\mathrm{F}_{2} \in \mathrm{R}^{\mathrm{t} \times \mathrm{r}}$ are arbitrary.

Proof. By Lemmas 2.2 and 2.4, Problem 1.3 is equivalent to find $A_{11} \in R^{(n-r) \times(n-r)}$ and $A_{22} \in R^{r \times r}$ such that

$$
A=D_{n}\left(\begin{array}{cc}
A_{11} & 0 \\
0 & A_{22}
\end{array}\right) D_{n}^{\top}
$$

where $A_{11}$ and $A_{22}$ must satisfy

$$
\begin{gathered}
A_{11} X_{1}=X_{1} \Lambda_{1}, \quad A_{22} Y_{1}=Y_{1} \Lambda_{2}, \\
A_{10}=A_{11}[k-t]=\left(0, I_{k-t}\right) A_{11}\left(0, I_{k-t}\right)^{\top}, \\
A_{20}=A_{22}[t]=\left(0, I_{t}\right) A_{22}\left(0, I_{t}\right)^{\top} .
\end{gathered}
$$

From Lemma 3.2, (3.11) holds if and only if

$$
X_{1} \Lambda_{1} X_{1}^{+} X_{1}=X_{1} \Lambda_{1}, \quad Y_{1} \Lambda_{2} Y_{1}^{+} Y_{1}=Y_{1} \Lambda_{2}
$$

which means that (3.7) holds. Moreover $A_{11}$ and $A_{22}$ can be written as

$$
A_{11}=X_{1} \Lambda_{1} X_{1}^{+}+G_{1}\left(I_{n-r}-X_{1} X_{1}^{+}\right), \quad A_{22}=Y_{1} \Lambda_{2} Y_{1}^{+}+G_{2}\left(I_{r}-Y_{1} Y_{1}^{+}\right),
$$

where $G_{1} \in R^{(n-r) \times(n-r)}$ and $G_{2} \in R^{r \times r}$ are arbitrary matrices. Substituting (3.13) into (3.12), and noticing (3.6), the definitions of $Z_{1}, Z_{2}, K_{1}$ and $K_{2}$, then $G_{1}$ and $G_{2}$ satisfy

$$
\left(0, I_{k-t}\right) G_{1} Z_{1}=K_{1}, \quad\left(0, I_{t}\right) G_{2} Z_{2}=K_{2} .
$$

Lemma 3.3 implies that (3.14) holds if and only if

$$
\left(0, I_{k-t}\right)\left(0, I_{k-t}\right)^{+} K_{1} Z_{1}^{+} Z_{1}=K_{1}, \quad\left(0, I_{t}\right)\left(0, I_{t}\right)^{+} K_{2} Z_{2}^{+} Z_{2}=K_{2} .
$$

We know from $\left(0, I_{k-t}\right)^{+}=\left(0, I_{k-t}\right)^{\top}$ and $\left(0, I_{t}\right)^{+}=\left(0, I_{t}\right)^{\top}$ that $\left(0, I_{k-t}\right)\left(0, I_{k-t}\right)^{+}=I_{k-t}$ and $\left(0, I_{t}\right)\left(0, I_{t}\right)^{+}=I_{t}$. Hence, (3.15) is equivalent to (3.8), and $G_{1}, G_{2}$ can be expressed as

$$
\begin{aligned}
G_{1} & =\left(0, I_{k-t}\right)^{+} K_{1} Z_{1}^{+}+\left(\begin{array}{l}
E_{1} \\
E_{2}
\end{array}\right)-\left(0, I_{k-t}\right)^{+}\left(0, I_{k-t}\right)\left(\begin{array}{l}
E_{1} \\
E_{2}
\end{array}\right) Z_{1} Z_{1}^{+} \\
& =\left(\begin{array}{c}
E_{1} \\
K_{1} Z_{1}^{+}+E_{2}\left(I_{n-r}-Z_{1} Z_{1}^{+}\right)
\end{array}\right), \quad \forall E_{1} \in R^{(n-r-k+t) \times(n-r)}, \quad \forall E_{2} \in R^{(k-t) \times(n-r),} \\
G_{2} & =\left(0, I_{t}\right)^{+} K_{2} Z_{2}^{+}+\left(\begin{array}{l}
F_{1} \\
F_{2}
\end{array}\right)-\left(0, I_{t}\right)^{+}\left(0, I_{t}\right)\left(\begin{array}{l}
F_{1} \\
F_{2}
\end{array}\right) Z_{2} Z_{2}^{+} \\
& =\left(\begin{array}{c}
F_{1} \\
K_{2} Z_{2}^{+}+F_{2}\left(I_{r}-Z_{2} Z_{2}^{+}\right)
\end{array}\right), \quad \forall F_{1} \in R^{(r-t) \times r}, \quad \forall F_{2} \in R^{t \times r} .
\end{aligned}
$$

Thus, the general solution to Problem 1.3 may be written as in (3.9). 


\section{The solution to Problem 1.4}

When the solution set of Problem 1.3 is nonempty, it is easy to verify that $S_{A}$ is a closed convex set, therefore there exists a unique solution $A^{*}$ to Problem 1.4. Now we give the expression for $A^{*}$.

Lemma 4.1. Given $\mathrm{X} \in \mathrm{R}^{\mathrm{n} \times \mathrm{m}}, \mathrm{I}_{\mathrm{n}}-\mathrm{XX}^{+}$and $\mathrm{XX}$ are orthogonal projection matrices, that is

$$
\left(I_{n}-X X^{+}\right)^{2}=I_{n}-X X^{+}=\left(I_{n}-X X^{+}\right)^{\top}, \quad\left(X X^{+}\right)^{2}=X X^{+}=\left(X X^{+}\right)^{\top} .
$$

Moreover, we have $\left(\mathrm{I}_{\mathrm{n}}-\mathrm{XX}^{+}\right) \mathrm{XX}^{+}=0$.

Lemma 4.1 can be verified easily upon computation.

Theorem 4.2. Let $X \in \mathrm{R}^{\mathrm{n} \times \mathrm{m}}$ form as in (3.1). Let $\Lambda \in \mathrm{R}^{\mathrm{m} \times \mathrm{m}}$ form as in (3.2). Given $\widetilde{A} \in \mathrm{R}^{\mathrm{n} \times \mathrm{n}}$ and $\mathrm{A}_{0} \in \mathrm{CSR}^{\mathrm{k} \times \mathrm{k}}$, denote

$$
D_{n}^{\top} \widetilde{A} D_{n}=\left(\begin{array}{ll}
\widetilde{A}_{11} & \widetilde{A}_{12} \\
\widetilde{A}_{21} & \widetilde{A}_{22}
\end{array}\right), \quad \widetilde{A}_{11} \in R^{(n-r) \times(n-r)}, \quad \widetilde{A}_{22} \in R^{r \times r},
$$

and partition $\widetilde{\mathrm{A}}_{11}$ and $\widetilde{\mathrm{A}}_{22}$ as

$$
\widetilde{A}_{11}=\left(\begin{array}{l}
P_{1} \\
P_{2}
\end{array}\right), \quad \widetilde{A}_{22}=\left(\begin{array}{l}
Q_{1} \\
Q_{2}
\end{array}\right),
$$

where $\mathrm{P}_{1} \in \mathrm{R}^{(\mathrm{n}-\mathrm{r}-\mathrm{k}+\mathrm{t}) \times(\mathrm{n}-\mathrm{r})}, \mathrm{P}_{2} \in \mathrm{R}^{(\mathrm{k}-\mathrm{t}) \times(\mathrm{n}-\mathrm{r})}, \mathrm{Q}_{1} \in \mathrm{R}^{(\mathrm{r}-\mathrm{t}) \times \mathrm{r}}$ and $\mathrm{Q}_{2} \in \mathrm{R}^{\mathrm{t} \times \mathrm{r}}$. Set

$$
\begin{aligned}
& \widetilde{\mathrm{P}}_{2}=\left(\mathrm{P}_{2}-\mathrm{K}_{1} \mathrm{Z}_{1}^{+}\right)\left(\mathrm{I}_{\mathrm{n}-\mathrm{r}}-\mathrm{X}_{1} \mathrm{X}_{1}^{+}\right), \quad \widetilde{\mathrm{Z}}_{1}=\left(\mathrm{I}_{\mathrm{n}-\mathrm{r}}-\mathrm{Z}_{1} \mathrm{Z}_{1}^{+}\right)\left(\mathrm{I}_{\mathrm{n}-\mathrm{r}}-\mathrm{X}_{1} \mathrm{X}_{1}^{+}\right), \\
& \widetilde{\mathrm{Q}}_{2}=\left(\mathrm{Q}_{2}-\mathrm{K}_{2} \mathrm{Z}_{2}^{+}\right)\left(\mathrm{I}_{\mathrm{r}}-\mathrm{Y}_{1} \mathrm{Y}_{1}^{+}\right), \quad \widetilde{\mathrm{Z}}_{2}=\left(\mathrm{I}_{\mathrm{r}}-\mathrm{Z}_{2} \mathrm{Z}_{2}^{+}\right)\left(\mathrm{I}_{\mathrm{r}}-\mathrm{Y}_{1} \mathrm{Y}_{1}^{+}\right) .
\end{aligned}
$$

If Problem 1.3 is solvable, then Problem 1.4 has a unique solution $A^{*}$, which can be written as

$$
A^{*}=D_{n}\left(\begin{array}{cc}
X_{1} \wedge_{1} X_{1}^{+}+\widetilde{G}_{1} & 0 \\
0 & Y_{1} \wedge_{2} Y_{1}^{+}+\widetilde{G}_{2}
\end{array}\right) D_{n}^{T}
$$

where $\widetilde{\mathrm{G}}_{1}=\left(\begin{array}{c}\mathrm{P}_{1}\left(\mathrm{I}_{\mathrm{n}-\mathrm{r}}-\mathrm{X}_{1} \mathrm{X}_{1}^{+}\right) \\ \mathrm{K}_{1} \mathrm{Z}_{1}^{+}\left(\mathrm{I}_{\mathrm{n}-\mathrm{r}}-\mathrm{X}_{1} \mathrm{X}_{1}^{+}\right)+\widetilde{\mathrm{P}}_{2} \widetilde{\mathrm{Z}}_{1}^{+} \widetilde{\mathrm{Z}}_{1}\end{array}\right)$, and $\widetilde{\mathrm{G}}_{2}=\left(\begin{array}{c}\mathrm{Q}_{1}\left(\mathrm{I}_{\mathrm{r}}-\mathrm{Y}_{1} \mathrm{Y}_{1}^{+}\right) \\ \mathrm{K}_{2} \mathrm{Z}_{2}^{+}\left(\mathrm{I}_{\mathrm{r}}-\mathrm{Y}_{1} \mathrm{Y}_{1}^{+}\right)+\widetilde{\mathrm{Q}}_{2} \widetilde{\mathrm{Z}}_{2}^{+} \widetilde{\mathrm{Z}}_{2}\end{array}\right)$.

Proof. Suppose that $A$ is an arbitrary solution to Problem 1.3, then by (3.9), we have

$$
\begin{aligned}
\|A-\widetilde{A}\|^{2}= & \left\|D_{n}\left(\begin{array}{cc}
X_{1} \wedge_{1} X_{1}^{+}+G_{1}\left(I_{n-r}-X_{1} X_{1}^{+}\right) & 0 \\
0 & Y_{1} \wedge_{2} Y_{1}^{+}+G_{2}\left(I_{r}-Y_{1} Y_{1}^{+}\right)
\end{array}\right) D_{n}^{\top}-\widetilde{A}\right\|^{2} \\
= & \left\|X_{1} \wedge_{1} X_{1}^{+}+G_{1}\left(I_{n-r}-X_{1} X_{1}^{+}\right)-\widetilde{A}_{11}\right\|^{2}+\left\|\widetilde{A}_{12}\right\|^{2} \\
& +\left\|\widetilde{A}_{21}\right\|^{2}+\left\|Y_{1} \wedge_{2} Y_{1}^{+}+G_{2}\left(I_{r}-Y_{1} Y_{1}^{+}\right)-\widetilde{A}_{22}\right\|^{2} .
\end{aligned}
$$

Hence $\|A-\widetilde{A}\|=\min _{A \in S_{A}}$ is equivalent to

$$
\begin{aligned}
\left\|G_{1}\left(I_{n-r}-X_{1} X_{1}^{+}\right)-\left(\widetilde{A}_{11}-X_{1} \Lambda_{1} X_{1}^{+}\right)\right\| & =\min _{\left.G_{1} \in R^{(n-r) \times(n-r)}\right)^{\prime}} \\
\left\|G_{2}\left(I_{r}-Y_{1} Y_{1}^{+}\right)-\left(\widetilde{A}_{22}-Y_{1} \Lambda_{2} Y_{1}^{+}\right)\right\| & =\min _{G_{2} \in R^{r \times r}} .
\end{aligned}
$$


Utilizing (3.10), (4.1), (4.2), and noticing Lemma 4.1, we get

$$
\begin{aligned}
&\left\|G_{1}\left(I_{n-r}-X_{1} X_{1}^{+}\right)-\left(\widetilde{A}_{11}-X_{1} \Lambda_{1} X_{1}^{+}\right)\right\|^{2} \\
&=\left\|\left[G_{1}\left(I_{n-r}-X_{1} X_{1}^{+}\right)-\left(\widetilde{A}_{11}-X_{1} \wedge_{1} X_{1}^{+}\right)\right]\left(I_{n-r}-X_{1} X_{1}^{+}\right)\right\|^{2} \\
& \quad+\left\|\left[G_{1}\left(I_{n-r}-X_{1} X_{1}^{+}\right)-\left(\widetilde{A}_{11}-X_{1} \wedge_{1} X_{1}^{+}\right)\right] X_{1} X_{1}^{+}\right\|^{2} \\
&=\left\|G_{1}\left(I_{n-r}-X_{1} X_{1}^{+}\right)-\widetilde{A}_{11}\left(I_{n-r}-X_{1} X_{1}^{+}\right)\right\|^{2}+\left\|\widetilde{A}_{11} X_{1} X_{1}^{+}-X_{1} \wedge_{1} X_{1}^{+}\right\|^{2} \\
&=\left\|\left(\begin{array}{c}
E_{1} Z_{1}^{+}+E_{2}\left(I_{n-r}-Z_{1} Z_{1}^{+}\right)
\end{array}\right)\left(I_{n-r}-X_{1} X_{1}^{+}\right)-\left(\begin{array}{c}
P_{1} \\
P_{2}
\end{array}\right)\left(I_{n-r}-X_{1} X_{1}^{+}\right)\right\|^{2} \\
&+\left\|\widetilde{A}_{11} X_{1} X_{1}^{+}-X_{1} \Lambda_{1} X_{1}^{+}\right\|^{2} \\
&=\left\|E_{1}\left(I_{n-r}-X_{1} X_{1}^{+}\right)-P_{1}\left(I_{n-r}-X_{1} X_{1}^{+}\right)\right\|^{2}+\left\|E_{2} \widetilde{Z}_{1}-\widetilde{P}_{2}\right\|^{2}+\left\|\widetilde{A}_{11} X_{1} X_{1}^{+}-X_{1} \Lambda_{1} X_{1}^{+}\right\|^{2} .
\end{aligned}
$$

Hence (4.4) is equivalent to

$$
\left\|E_{1}\left(I_{n-r}-X_{1} X_{1}^{+}\right)-P_{1}\left(I_{n-r}-X_{1} X_{1}^{+}\right)\right\|=\min , \quad\left\|E_{2} \widetilde{Z}_{1}-\widetilde{P}_{2}\right\|=\min .
$$

We know from Lemma 3.4 that (4.6) holds, which implies that (4.4) holds, if and only if

$$
E_{1}=P_{1}\left(I_{n-r}-X_{1} X_{1}^{+}\right)+\widetilde{E}_{1} X_{1} X_{1}^{+}, \quad E_{2}=\widetilde{P}_{2} \widetilde{Z}_{1}^{+}+\widetilde{E}_{2}\left(I_{n-r}-\widetilde{Z}_{1} \widetilde{Z}_{1}^{+}\right),
$$

where $\widetilde{E}_{1} \in R^{(n-r-k+t) \times(n-r)}$ and $\widetilde{E}_{2} \in R^{(k-t) \times(n-r)}$ are arbitrary matrices.

We can prove in a similar way that (4.5) holds if and only if

$$
\begin{aligned}
& F_{1}=Q_{1}\left(I_{r}-Y_{1} Y_{1}^{+}\right)+\widetilde{F}_{1} Y_{1} Y_{1}^{+}, \\
& F_{2}=\widetilde{Q}_{2} \widetilde{Z}_{2}^{+}+\widetilde{F}_{2}\left(I_{r}-\widetilde{Z}_{2} \widetilde{Z}_{2}^{+}\right),
\end{aligned}
$$

where $\widetilde{F}_{1} \in R^{(r-t) \times r}$ and $\widetilde{F}_{2} \in R^{t \times r}$ are arbitrary matrices.

Substituting $E_{1}, E_{2}$ and $F_{1}, F_{2}$ into (3.10), we get that the unique solution to Problem 1.4 can be expressed as in (4.3) as desired.

\section{Algorithm 4.3.}

(1) Input $X \in R^{n \times m}$ as in (3.1), $\Lambda \in R^{m \times m}$ as in (3.2), $\widetilde{A} \in R^{n \times n}$ and $A_{0} \in C R^{k \times k}$.

(2) Partition $A_{0}$ as in (2.9) to get $A_{10}$ and $A_{20}$.

(3) Obtain $X_{1}$ and $Y_{1}$ according to (3.3).

(4) Follow (3.6) to calculate $Z_{1}, Z_{2}, K_{1}$ and $K_{2}$.

(5) If (3.7) and (3.8) hold, then continue; otherwise stop.

(6) According to Theorem 4.2 calculate $\widetilde{A}_{11}, \widetilde{A}_{22}, \mathrm{P}_{1}, \mathrm{Q}_{1}, \widetilde{\mathrm{P}}_{2}, \widetilde{\mathrm{Z}}_{1}, \widetilde{\mathrm{Q}}_{2}, \widetilde{\mathrm{Z}}_{2}$ and $A^{*}$.

Exemple 4.4. Assume $\mathrm{n}=10, \mathrm{k}=4, \mathrm{~m}=4$. Given

$$
\Lambda=\left(\begin{array}{cccc}
-2.7645 & 0 & 0 & 0 \\
0 & 0.8744 & 0 & 0 \\
0 & 0 & -2.8382 & 0 \\
0 & 0 & 0 & -1.3716
\end{array}\right), \quad A_{0}=\left(\begin{array}{cccc}
-1.65 & 0.55 & 0.55 & 0.25 \\
0.35 & -0.6 & -0.2 & 0.45 \\
0.45 & -0.2 & -0.6 & 0.35 \\
0.25 & 0.55 & 0.55 & -1.65
\end{array}\right) \text {, }
$$




$$
\begin{aligned}
& X=\left(\begin{array}{cccc}
-0.1371 & 0.2076 & -0.2167 & 0.1560 \\
-0.1338 & 0.4773 & -0.0028 & 0.4014 \\
0.0780 & 0.1427 & 0.4728 & 0.0788 \\
-0.5429 & 0.0952 & -0.4434 & 0.3097 \\
0.4030 & 0.4469 & -0.1812 & -0.4609 \\
0.4030 & 0.4469 & 0.1812 & 0.4609 \\
-0.5429 & 0.0952 & 0.4434 & -0.3097 \\
0.0780 & 0.1427 & -0.4728 & -0.0788 \\
-0.1338 & 0.4773 & 0.0028 & -0.4014 \\
-0.1371 & 0.2076 & 0.2167 & -0.1560
\end{array}\right) \\
& \widetilde{A}=\left(\begin{array}{cccccccccc}
1.7643 & -0.6475 & 0.7996 & -1.4946 & -1.0485 & 0.2050 & -0.2997 & 0.3591 & 1.1636 & 0.1017 \\
0.9457 & 0.8022 & -0.4446 & -0.7732 & 1.3888 & -0.5330 & 0.2510 & -0.9312 & -0.0488 & -0.5711 \\
-0.2314 & -0.1201 & 0.1656 & 1.1546 & 0.4174 & -0.3023 & -0.3856 & 1.5166 & -0.1959 & 0.4041 \\
-0.0590 & -0.2892 & 1.2945 & -1.5725 & 0.6320 & 0.5720 & 0.3005 & 0.0652 & -0.1688 & -0.8455 \\
-0.5931 & 1.8189 & -0.5775 & 0.4104 & -0.5819 & -0.1916 & 0.5068 & -0.8231 & 0.1822 & 0.6014 \\
0.5694 & 0.2275 & -0.8018 & 0.4816 & -0.1903 & -0.5641 & 0.4142 & -0.5604 & 1.8448 & -0.6054 \\
-0.7796 & -0.1209 & 0.0171 & 0.2933 & 0.5531 & 0.6233 & -1.6293 & 1.2396 & -0.2793 & -0.1009 \\
0.4152 & -0.1291 & 1.5941 & -0.3157 & -0.3990 & 0.4602 & 1.1340 & 0.1692 & -0.1511 & -0.1840 \\
-0.6168 & -0.0053 & -0.9043 & 0.2626 & -0.5033 & 1.3451 & -0.7673 & -0.4762 & 0.8445 & 0.9005 \\
0.1522 & 1.1798 & 0.3175 & -0.2727 & 0.2888 & -1.0087 & -1.4385 & 0.7949 & -0.5679 & 1.7742
\end{array}\right),
\end{aligned}
$$

then we can obtain the best approximate solution $A^{*}$ to Problem 1.4 by Algorithm 4.3, where

$$
A^{*}=\left(\begin{array}{cccccccccc}
1.7418 & -0.6746 & 0.7747 & -1.4808 & -1.0768 & 0.1823 & -0.2974 & 0.3248 & 1.1168 & 0.1012 \\
0.8863 & 0.7497 & -0.4751 & -0.7970 & 1.2891 & -0.5704 & 0.2258 & -0.9470 & -0.1194 & -0.6331 \\
-0.2421 & -0.2142 & 0.1429 & 1.1080 & 0.4041 & -0.4036 & -0.3916 & 1.5472 & -0.2300 & 0.3731 \\
-0.0915 & -0.3337 & 1.2344 & -1.6500 & 0.5500 & 0.5500 & 0.2500 & 0.0143 & -0.2126 & -0.8356 \\
-0.6369 & 1.7404 & -0.5895 & 0.3500 & -0.6000 & -0.2000 & 0.4500 & -0.8821 & 0.1326 & 0.5793 \\
0.5793 & 0.1326 & -0.8821 & 0.4500 & -0.2000 & -0.6000 & 0.3500 & -0.5895 & 1.7404 & -0.6369 \\
-0.8356 & -0.2126 & 0.0143 & 0.2500 & 0.5500 & 0.5500 & -1.6500 & 1.2344 & -0.3337 & -0.0915 \\
0.3731 & -0.2300 & 1.5472 & -0.3916 & -0.4036 & 0.4041 & 1.1080 & 0.1429 & -0.2142 & -0.2421 \\
-0.6331 & -0.1194 & -0.9470 & 0.2258 & -0.5704 & 1.2891 & -0.7970 & -0.4751 & 0.7497 & 0.8863 \\
0.1012 & 1.1168 & 0.3248 & -0.2974 & 0.1823 & -1.0768 & -1.4808 & 0.7747 & -0.6746 & 1.7418
\end{array}\right) .
$$

Exemple 4.5. Assume $n=10, k=4, m=5$. Let $A_{0}$ be the same matrix as in Example 4.4, and

$$
\begin{aligned}
& \Lambda=\left(\begin{array}{ccccc}
-2.7645 & 0 & 0 & 0 & 0 \\
0 & 0.8744 & 0 & 0 & 0 \\
0 & 0 & 1.6888 & 0.6148 & 0 \\
0 & 0 & -0.6148 & -1.6888 & 0 \\
0 & 0 & 0 & 0 & -1.3716
\end{array}\right), X=\left(\begin{array}{cccccc}
-0.1371 & 0.2076 & -0.5872 & 0.0000 & 0.1560 \\
-0.1338 & 0.4773 & -0.1075 & 0.1681 & 0.4014 \\
0.0780 & 0.1427 & 0.1565 & -0.0217 & 0.0788 \\
-0.5429 & 0.0952 & -0.0595 & -0.0017 & 0.3097 \\
0.4030 & 0.4469 & 0.2920 & 0.0398 & -0.4609 \\
0.4030 & 0.4469 & -0.2920 & -0.0398 & 0.4609 \\
-0.5429 & 0.0952 & 0.0595 & 0.0017 & -0.3097 \\
0.0780 & 0.1427 & -0.1565 & 0.0217 & -0.0788 \\
-0.1338 & 0.4773 & 0.1075 & -0.1681 & -0.4014 \\
-0.1371 & 0.2076 & 0.5872 & 0 & -0.1560
\end{array}\right) \\
& \widetilde{A}=\left(\begin{array}{cccccccccc}
1.7572 & -0.6165 & 0.7680 & -1.4552 & -1.0340 & 0.2842 & -0.2699 & 0.3469 & 1.1695 & 0.1680 \\
0.9292 & 0.8229 & -0.4083 & -0.7349 & 1.3295 & -0.5869 & 0.2496 & -0.9913 & -0.0295 & -0.5985 \\
-0.2080 & -0.1178 & 0.1257 & 1.1170 & 0.4950 & -0.3811 & -0.3742 & 1.5829 & -0.1819 & 0.4022 \\
-0.0967 & -0.2652 & 1.2886 & -1.5969 & 0.6194 & 0.5654 & 0.3233 & 0.0686 & -0.1478 & -0.8397 \\
-0.5574 & 1.7665 & -0.5080 & 0.4134 & -0.5793 & -0.1971 & 0.4617 & -0.8733 & 0.1796 & 0.6497 \\
0.6399 & 0.1528 & -0.8700 & 0.4514 & -0.1445 & -0.5991 & 0.4246 & -0.5031 & 1.7963 & -0.6141 \\
-0.7955 & -0.1045 & 0.0073 & 0.2970 & 0.6379 & 0.6096 & -1.5690 & 1.2184 & -0.2075 & -0.0875 \\
0.4401 & -0.1320 & 1.5767 & -0.3114 & -0.3442 & 0.4609 & 1.1745 & 0.1300 & -0.1784 & -0.2107 \\
-0.6448 & -0.0139 & -0.9915 & 0.2114 & -0.5248 & 1.3919 & -0.7663 & -0.4589 & 0.8001 & 0.8508 \\
0.1809 & 1.2439 & 0.3729 & -0.2557 & 0.2895 & -1.0266 & -1.4416 & 0.7236 & -0.5593 & 1.7545
\end{array}\right) .
\end{aligned}
$$


In this case, $\widetilde{Z}_{2}=0$, thus we can simplify $\widetilde{G}_{2}=\left(\begin{array}{c}\mathrm{Q}_{1}\left(\mathrm{I}_{\mathrm{r}}-\mathrm{Y}_{1} \mathrm{Y}_{1}^{+}\right) \\ \mathrm{K}_{2} \mathrm{Z}_{2}^{+}\left(\mathrm{I}_{\mathrm{r}}-\mathrm{Y}_{1} \mathrm{Y}_{1}^{+}\right)+\widetilde{\mathrm{Q}}_{2} \widetilde{\mathrm{Z}}_{2}^{+} \widetilde{\mathrm{Z}}_{2}\end{array}\right)$ in Theorem 4.2 to $\widetilde{\mathrm{G}}_{2}=$ $\left(\begin{array}{c}Q_{1} \\ K_{2} Z_{2}^{+}\end{array}\right)\left(I_{r}-Y_{1} Y_{1}^{+}\right)$, and the unique solution $A^{*}$ to Problem 1.4 is

$$
A^{*}=\left(\begin{array}{cccccccccc}
1.7162 & -0.6778 & 0.7252 & -1.4836 & -1.1027 & 0.2208 & -0.2919 & 0.3335 & 1.1176 & 0.1318 \\
0.8660 & 0.7810 & -0.4566 & -0.7759 & 1.3222 & -0.6060 & 0.2012 & -0.9996 & -0.1181 & -0.6573 \\
-0.2321 & -0.2322 & 0.1201 & 1.0968 & 0.4102 & -0.3935 & -0.3691 & 1.5516 & -0.2269 & 0.3700 \\
-0.1327 & -0.3110 & 1.2116 & -1.6500 & 0.5500 & 0.5500 & 0.2500 & 0.0116 & -0.2110 & -0.8327 \\
-0.5930 & 1.7138 & -0.5619 & 0.3500 & -0.6000 & -0.2000 & 0.4500 & -0.8619 & 0.1138 & 0.6070 \\
0.6070 & 0.1138 & -0.8619 & 0.4500 & -0.2000 & -0.6000 & 0.3500 & -0.5619 & 1.7138 & -0.5930 \\
-0.8327 & -0.2110 & 0.0116 & 0.2500 & 0.5500 & 0.5500 & -1.6500 & 1.2116 & -0.3110 & -0.1327 \\
0.3700 & -0.2269 & 1.5516 & -0.3691 & -0.3935 & 0.4102 & 1.0968 & 0.1201 & -0.2322 & -0.2321 \\
-0.6573 & -0.1181 & -0.9996 & 0.2012 & -0.6060 & 1.3222 & -0.7759 & -0.4566 & 0.7810 & 0.8660 \\
0.1318 & 1.1176 & 0.3335 & -0.2919 & 0.2208 & -1.1027 & -1.4836 & 0.7252 & -0.6778 & 1.7162
\end{array}\right) .
$$

\section{Acknowledgment}

The authors would like to thank Prof. Xiao-Jun Yang and the referees very much for their valuable suggestions and comments, which have greatly improved this article. This research was supported by Scientific Research Fund of Zhejiang Provincial Education Department (Y201738285).

\section{References}

[1] A. L. Andrew, Eigenvectors of certain matrices, Linear Algebra and Appl., 7 (1973), 157-162. 1

[2] A. Borobia, R. Canogar, The real nonnegative inverse eigenvalue problem is NP-hard, Linear Algebra Appl., 522 (2017), 127-139. 1

[3] M. T. Chu, Inverse eigenvalue problems, SIAM Rev., 40 (1998), 1-39.

[4] L.-F. Dai, M.-L. Liang, W.-Y. Ma, Optimization problems on the rank of the solution to left and right inverse eigenvalue problem, J. Ind. Manag. Optim., 11 (2015), 171-183. 1

[5] L. Datta, S. D. Morgera, On the reducibility of centrosymmetric matrices-applications in engineering problems, Circuits Systems Signal Process., 8 (1989), 71-96. 1

[6] P. Deift, T. Nanda, On the determination of a tridiagonal matrix from its spectrum and a submatrix, Linear Algebra Appl., 60 (1984), 43-55. 1

[7] G. H. Golub, C. F. Van Loan, Matrix computations, Third edition, Johns Hopkins Studies in the Mathematical Sciences, Johns Hopkins University Press, Baltimore, MD, (1996). 2.1

[8] I. J. Good, The inverse of a centrosymmetric matrix, Technometrics, 12 (1970), 925-928. 1

[9] X. Y. Hu, L. Zhang, Least-square approximation solutions to a class of matrix problems, (Chinese) Hunan Daxue Xuebao, 17 (1990), 98-102. 3.3

[10] Z. X. Jiang, Q. S. Lu, On optimal approximation of a matrix under a spectral restriction, (Chinese) Math. Numer. Sinica, 8 (1986), 47-52. 1, 3.2

[11] K. T. Joseph, Inverse eigenvalue problem in structural design, AIAA J., 30 (1992), 2890-2896. 1

[12] M. Kimura, Some problems of stochastic processes in genetics, Ann. Math. Statist., 28 (1957), 882-901. 1

[13] N. Li, A matrix inverse eigenvalue problem and its application, Linear Algebra Appl., 266 (1997), 143-152. 1

[14] Z.-Y. Peng, X.-Y. Hu, L. Zhang, The inverse problem of centrosymmetric matrices with a submatrix constraint, J. Comput. Math., 22 (2004), 535-544. 1

[15] Y. M. Ram, J. Caldwell, Physical parameters reconstruction of a free-free mass-spring system from its spectra, SIAM J. Appl. Math., 52 (1992), 140-152. 1

[16] J. R. Weaver, Centrosymmetric (cross-symmetric) matrices, their basic properties, eigenvalues, and eigenvectors, Amer. Math. Monthly, 92 (1985), 711-717. 1

[17] J. Xu, D. X. Xie, Central symmetric least squares solution for matrix equation with a submatrix constraint, J. B. Inf. Sci. Technol. Univ., 31 (2016), 31-35. 1

[18] Q.-X. Yin, Construction of real antisymmetric and bi-antisymmetric matrices with prescribed spectrum data, Linear Algebra Appl., 389 (2004), 95-106. 1

[19] L. A. Zhornitskaya, V. S. Serov, Inverse eigenvalue problems for a singular Sturm-Liouville operator on [0,1], Inverse Problems, 10 (1994), 975-987. 1

[20] F.-Z. Zhou, X.-Y. Hu, L. Zhang, The solvability conditions for the inverse eigenvalue problems of centro-symmetric matrices, Linear Algebra Appl., 364 (2003), 147-160. 1, 1, 2.2 